\section{BETA: A FORTRAN IV program for calculating the mean, standard deviation, and the mode of the prior and posterior beta distributions}

\author{
AMNON TILL \\ Victoria University of Wellington \\ Private Bag, Wellington, New Zealand
}

In Bayesian statistics the beta distribution is frequently used when one wants to make inferences about proportions. The general form of the beta distribution is

$$
y=\frac{(p+q-1) !}{(p-1) !(q-1) !} \Pi^{p-1}(1-\Pi)^{q-1}
$$

where $y$ is the probability density, $\Pi$ is the proportion, and $p$ and $q$ are the parameters of the equation. The values of $p$ and $q$ must be greater than zero.

According to Bayesian statistics (Phillips, 1973), if the prior distribution is a beta and if the data are obtained by making successive independent observations, on which only one of two events can occur, then the posterior distribution will be beta.

For example, one can try to estimate the proportion of red chips in an urn containing red and blue chips. If the true proportion of red chips in the urn is defined as $\Pi$, then $\Pi^{*}$ is the estimated proportion of red chips in the sample.

One of the beta distributions described by Phillips (1973, Appendix B) can be used to describe the prior distribution of $\Pi^{*}$. Then, using $p^{\prime}$ and $q^{\prime}$ as the parameter of the prior distribution, one can calculate the mean $\left(\mathrm{m}^{\prime}\right)$, standard deviation $\left(\mathrm{s}^{\prime}\right)$, and mode $\left(\mathrm{mo}^{\prime}\right)$ of that distribution.

The calculations can be done in the following way:

$$
\begin{aligned}
\mathrm{m}^{\prime} & =\frac{\mathrm{p}^{\prime}}{\mathrm{p}^{\prime}+\mathrm{q}^{\prime}} \\
\mathrm{s}^{\prime} & =\sqrt{\frac{\mathrm{p}^{\prime} \mathrm{q}^{\prime}}{\left(\mathrm{p}^{\prime}+\mathrm{q}^{\prime}\right)^{2}\left(\mathrm{p}^{\prime}+\mathrm{q}^{\prime}+1\right)}} \\
\mathrm{mo}^{\prime} & =\frac{\mathrm{p}^{\prime}-1}{\mathrm{p}^{\prime}+\mathrm{q}^{\prime}-2}
\end{aligned}
$$

Then the $95 \%$ or $99 \%$ interval for the highest density regions of the prior distribution can be found by looking at Appendix B of Phillips (1973), at the appropriate $p$ and $q$ values.
After calculating the values of the prior distribution of the proportion of red chips in the urn, one can take a random sample of chips, with replacement, from the urn. Each observation of a red chip can be recorded as a "success" (s) and each observation of a blue chip as a "failure" (f). If $\mathrm{N}$ observations are taken, then $\mathrm{N}=\overline{\mathrm{s}}+\overline{\mathrm{f}}$, where $\overline{\mathrm{s}}$ and $\overline{\mathrm{f}}$, respectively, stand for the number of successes and the number of failures. Now, the new posterior distribution of opinion will also be a beta with parameters $\mathrm{p}^{\prime \prime}$ and $\mathrm{q}^{\prime \prime}$, where

$$
\begin{aligned}
& \mathrm{p}^{\prime \prime}=\mathrm{p}^{\prime}+\overline{\mathrm{s}} \\
& \mathrm{q}^{\prime \prime}=\mathrm{q}^{\prime}+\overline{\mathrm{f}} .
\end{aligned}
$$

The mean $\left(\mathrm{m}^{\prime \prime}\right)$, standard deviation $\left(\mathrm{s}^{\prime \prime}\right)$, and mode (mo") of the posterior distribution of red chips in the urn can then be calculated in the same way as for the prior distribution. (See Equations 2, 3, and 4.)

$$
\begin{aligned}
\mathrm{m}^{\prime \prime} & =\frac{\mathrm{p}^{\prime \prime}}{\mathrm{p}^{\prime \prime}+\mathrm{q}^{\prime \prime}} \\
\mathrm{s}^{\prime \prime} & =\sqrt{\frac{\mathrm{p}^{\prime \prime} \mathrm{q}^{\prime \prime}}{\left(\mathrm{p}^{\prime \prime}+\mathrm{q}^{\prime \prime}\right)^{2}\left(\mathrm{p}^{\prime \prime}+\mathrm{q}^{\prime \prime}+1\right)}} \\
\mathrm{mo}^{\prime \prime} & =\frac{\mathrm{p}^{\prime \prime}-1}{\mathrm{p}^{\prime \prime}+\mathrm{q}^{\prime \prime}-2}
\end{aligned}
$$

Again, as for the prior distribution, we can get the 95\% and $99 \%$ intervals for the highest density regions of the posterior distribution by looking at Appendix B of Phillips (1973) at the appropriate $p$ and $q$ values.

The Program. BETA is a program designed to facilitate the calculation of the various statistics of the beta distribution. It can be used in several ways: (1) for teaching students this type of task, (2) as a subroutine in a bigger program that may provide the subjects with feedback on the various normative solutions to the task, and (3) as a tool for generating research hypotheses.

Input. The program varies (1) the values of the parameters of the prior beta distributions, $p^{\prime}$ and $q^{\prime}$ and (2) the number of "successes" $(\bar{s})$ and "failures" (f) in the sample.

Output. The program calculates (1) the mean $\left(\mathrm{m}^{\prime}\right)$, standard deviation $\left(\mathrm{s}^{\prime}\right)$, and mode $\left(\mathrm{mo}^{\prime}\right)$ of the prior distribution; (2) the value of the parameters of the posterior probability beta distributions ( $\mathrm{p}^{\prime \prime}$ and $\mathrm{q}^{\prime \prime}$ ); (3) the mean $\left(\mathrm{m}^{\prime \prime}\right)$, standard deviation $\left(\mathrm{s}^{\prime \prime}\right)$, and mode (mo") of the posterior probability distribution.

Restrictions. The program is written in FORTRAN IV, so any computer system that supports FORTRAN IV can run it. Program run time is $3.9 \mathrm{sec}$ on the Burroughs B6700 computer. 
544 TILL

Availability. A copy of the listing, documentation,

\section{REFERENCE}

and sample of output can be obtained free of charge by writing to Amnon Till, Department of Psychology, Victoria University of Wellington, Private Bag, Wellington, New Zealand.

Phillips. L. D. Bayesian statistics for social scientists, London: Nelson. 1973 .

(Accepted for publication June 21, 1977.) 